Ein neuer Rahmen für die Klima- und Energiepolitik in der EU

\section{Ehrgeiziger als der Ruf}

\author{
Im Januar hat die Europäische Kommission die Architektur \\ für die Klima- und Energiepolitik von 2020 bis 2030 \\ vorgeschlagen. Die Kritik in den deutschen Medien ist \\ ausgeprägt - ist sie auch gerechtfertigt? Ein genauerer \\ Blick auf die vorgeschlagenen Ziele lohnt sich. \\ Von Jan Nill
}

$D^{i}$ ie Europäische Union (EU) ist auf einem guten Weg, die für die Senkung der Treibhausgasemissionen und für den Ausbau der erneuerbaren Energien bis 2020 festgelegten 20-ProzentZiele zu erreichen.

Die Kommission schlägt als Kernstück der EU-Energie- und Klimapolitik bis 2030 [1] eine neue Zielvorgabe für die Minderung der EU-internen Treibhausgasemissionen von 40 Prozent gegenüber dem Stand von 1990 vor. Diese Zielvorgabe muss von den EU-Emissionshandels-Sektoren, einschließlich des internationalen Flugverkehrs, und den nicht vom EU-Emissionshandel erfassten Sektoren gemeinsam und auf kosteneffiziente Weise erreicht werden. Anders als beim 20-Prozent-Emissionsreduktionsziel für 2020 können auf dieses Ziel keine internationalen Emissionsgutschriften angerechnet werden. Und das Ziel ist an keine Bedingungen im Hinblick auf die internationalen Klimaverhandlungen geknüpft. Sollte das Ergebnis der internationalen Verhandlungen ein ehrgeizigeres Klimaziel für die Union rechtfertigen, könnten diese zusätzlichen Anstrengungen dadurch ausgeglichen werden, dass der Zugang zu internationalen Gutschriften ermöglicht wird.

Ist dieses Reduktionssziel nicht ehrgeizig genug, wie vielfach kritisiert wurde? Erstens ist das Ziel vollständig mit dem EU-2050-Ziel und den EU-Fahrplänen zur Erreichung von mindestens 80 Prozent interner Emissionsreduzierung kohärent. Zweitens sind bis 2012 die Treibhausgasemissionen in 22 Jahren um 18 Prozent zurückgegangen, wozu auch Wirtschaftskrisen ein Stück weit beigetragen haben. Gefordert wird also eine Erhöhung des Reduktionstempos auf 22 Prozent in 18 Jahren, also mehr als ein Prozent im Jahr. Drittens ist zwar richtig, dass bereits aufgrund der aktuellen politischen Maßnahmen die Emissionen der EU im Vergleich zum Niveau von 1990 bis 2020 um 24 Prozent und bis 2030 um 32 Prozent sinken könnten. Allerdings rechnet dies bereits die Folgen einer Reihe von ehrgeizigen Politiken mit ein, etwa des Emissionshandels mit bereits bestehendem jährlichen Reduktionsfaktor von 1,74 Prozent, die neue Energieeffizienzrichtlinie sowie eine Senkung des $\mathrm{CO}_{2}$-Standards für Fahrzeuge auf $95 \mathrm{Gramm} \mathrm{CO}_{2}$ pro Kilometer.

\section{Anspruchsvolle Ziele}

Um das -40-Prozent-Ziel zu erreichen, müssten die vom Emissionshandel erfassten Sektoren bis 2030 eine Minderung um 43 Prozent und die Sektoren außerhalb des Emissionshandels eine Verringerung um 30 Prozent (jeweils gegenüber dem Stand von 2005) erzielen. Die Zielvorgabe für letztere würde auf die Mitgliedstaaten aufgeteilt werden. Für Deutschland würde eine kosteneffektive Aufteilung bedeuten, dass dieses Ziel von derzeit-14 Prozent bis 2020 auf -41 Prozent bis 2030 ansteigen würde. Dabei ist aber auch eine faire Lastenteilung zwischen den Mitgliedstaaten wichtig, die deren spezifischen Gegebenheiten und Kapazitäten Rechnung trägt. Die Analyse zeigt beispielsweise, dass Länder mit einem Bruttoinlandsprodukt von weniger als 90 Prozent des EU-Durchschnitts im Zeitraum von 2021 bis 2030 Investitionen tätigen müssten, die rund drei Milliarden Euro pro Jahr höher sind als der Anstieg des EU-Durchschnitts im selben Zeitraum.

\section{Förderliches Umfeld für die Energiewende}

Weiter wird ein kohärentes und verbindliches Ziel für erneuerbare Energien auf europäischer Ebene von mindestens 27 Prozent des Endenergieverbrauchs vorgeschlagen. Deutschland würde wie alle Mitgliedstaaten über die Flexibilität verfügen, nationale Ziele festzulegen. Die Höhe des EU-Ziels ist kohärent mit einer kosteneffizienten Erreichung des Emissionsziels von -40 Prozent. Im Stromsektor entspricht dies einem EU-weiten Erneuerbaren-Anteil von mindestens 45 Prozent. Es würde durch eindeutige, von den Mitgliedstaaten selbst beschlossene Verpflichtungen im Einklang mit dem EU-Ziel erreicht werden. Dies würde im Rahmen eines neuen Governance-Prozesses überprüft. Es soll, soweit erforderlich, durch weitere EU-Maßnahmen und -Instrumente ergänzt werden, damit das Ziel erreicht wird.

Der Europäische Rat der Regierungschefs wird sich im März mit dem Politikrahmen befassen. Dann wird sich erweisen, ob der Kommissionsvorschlag nicht nur ehrgeizig, sondern auch realistisch ist.

\section{Anmerkung}

[1] Europäische Kommission: Ein Rahmen für die Klima- und Energiepolitik im Zeitraum 2020-2030. Brüssel, KOM (2014) 15. Im Internet: http://ec.europa.eu/clima/policies/2030.

AUTOR

Dr.Jan Nill ist Referent für die ökonomische Analyse von EU Klima- und Energiepolitiken in der Europäischen Kommission.

Europäische Kommission, GD Klimapolitik, Referat CLIMA A4. Tel.: +32 22990190 E-Mail: Jan.Nill@ec.europa.eu 